\title{
PDL1 blockage increases fetal resorption and Tfr cells but does not affect Th/Tfr ratio and B-cell maturation during allogeneic pregnancy
}

Weihong Zeng', Shi Qin', Renjie Wang², Yuchen Zhang ${ }^{1}$, Xiaoling Ma', Fuju Tian', Xiao-Rui Liu', Xiaoli Qin', Shujie Liao ${ }^{2}$, Liqun Sun ${ }^{1}$ and Yi Lin ${ }^{1}$

\begin{abstract}
A successful pregnancy requires sophisticated regulation of uterine microenvironment to guarantee the existence of semi-allogeneic conceptus without immune rejection. T follicular regulatory (Tfr) cells exert a suppressive effect on Tfh-cell expansion, B-cell response, and antibody production. Although accumulating evidence has demonstrated that dysregulations of Tfr cells can bring on various immunological diseases, their immunomodulatory roles during pregnancy still remain unheeded. Herein, we introduced an allogeneic normalpregnant mouse model and found that $C D 4^{+} \mathrm{CXCR} 5^{\text {hiPD }}-1^{\text {hi }} \mathrm{Foxp} 3^{+} \mathrm{Tfr}$ cells were preferentially accumulated in the uterus at mid-gestation and displayed a distinct phenotype. In addition, the absence of PDL1 resulted in increased fetal resorption by favoring Tfr cells accumulation and upregulating PD-1 expression on these cells. However, PDL1 blockade affected neither the ratio of Tfh/Tfr cells nor the maturation and differentiation of B cells. Overall, our results are the first to present a correlation of Tfr cells accumulation with healthy allogeneic pregnancy and PDL1 blockade-induced miscarriage, and to indicate that appropriate assembly of Tfr cells is important for pregnancy maintenance. Since blockade of PD-1-PDL1 pathway leads to more Tfr cells and fetal losses, the reproductive safety must be taken into consideration when PD-1/PD-L1 checkpoint blockade immunotherapy is used in pregnancy.
\end{abstract}

\section{Introduction}

A successful pregnancy requires extremely sophisticated regulation of uterine microenvironment to guarantee the existence of semi-allogeneic conceptus without immune rejection. Orchestrated balance of immune cells residing at the maternal-fetal interface fundamentally contributes to the immune defense protecting the host from external threats, as well as the maintenance of immune tolerance

\footnotetext{
Correspondence: Yi Lin (yilinonline@126.com) or Liqun Sun (slq.cn@163.com) or Shujie Liao (sjliao@tjh.tjmu.edu.cn)

'Shanghai Key Laboratory of Embryo Original Diseases, The International Peace Maternity \& Child Health Hospital, Shanghai Jiao Tong University School of Medicine, Shanghai 200030, P. R. China

${ }^{2}$ Tongji Hospital, Tongji Medical College, Huazhong University of Science and Technology, Wuhan 430030 Hubei, P. R. China

These authors contributed equally: Weihong Zeng, Shi Qin

Edited by H.U. Simon
}

keeping the fetal from maternal immune attack $^{1,2}$. An in-depth exploration into the unique immunological alterations during pregnancy will partially shed light on the pathogenesis of pregnancy-related complications such as spontaneous abortion and preterm delivery, and provide better strategies to improve perinatal outcomes and offspring's health ${ }^{3,4}$.

Programmed cell death 1 (PD-1) is well known as an inhibitory transmembrane receptor belonging to the B7CD28 family and expresses on various activated immune cells, especially activated $\mathrm{T}$ cells $\mathrm{s}^{5,6}$. The engagement of PD-1 and its prior binding ligand programmed death ligand-1 (PDL1) recruits second signals required for T cell exhaustion that are characterized by reduced proliferation, diminished cytokine production, and functionally silenced cytotoxic effector, serving as a negative

\section{(c) The Author(s) 2020}

(c) (i) Open Access This article is licensed under a Creative Commons Attribution 4.0 International License, which permits use, sharing, adaptation, distribution and reproduction in any medium or format, as long as you give appropriate credit to the original author(s) and the source, provide a link to the Creative Commons license, and indicate if changes were made. The images or other third party material in this article are included in the article's Creative Commons license, unless indicated otherwise in a credit line to the material. If material is not included in the article's Creative Commons license and your intended use is not permitted by statutory regulation or exceeds the permitted use, you will need to obtain permission directly from the copyright holder. To view a copy of this license, visit http://creativecommons.org/licenses/by/4.0/. 
checkpoint for proper immune responses ${ }^{7,8}$. Several reports have proposed the importance of PD-1-PDL1 interaction involved in pregnancy maintenance, and increased PD-1 expression was detected in decidual immune cells in the first trimester of normal pregnancy ${ }^{9,10}$. In addition, previous studies have established links among PD-1 and PDL1 expression, Th1/Th2/Th17 balance and fetomaternal tolerance ${ }^{11,12}$. A deficiency of PDL1 leads to a reduced ratio of regulatory T/effector $\mathrm{T}$ cells (Treg/Teff) and drives a shift toward Th1- and Th17-cell expansion, which represents a barrier to induce tolerance, resulting in increased embryo resorption and miscarriage ${ }^{11,12}$. Our previous study has revealed that the reduction of fetal survival rate induced by PDL1 blockade is associated with enhanced accumulation of $\mathrm{T}$ follicular helper (Tfh) cells and upregulated expression of ICOS and PD-1, developing the Th1/Th2/Th17/Treg paradigm into a Th1/Th2/Th17/Treg/Tfh balance in the formation and maintenance of maternal-fetal tolerance during the healthy pregnancy ${ }^{13}$.

As a novel and specialized subset of $\mathrm{CD}^{+}{ }^{+} \mathrm{T}$ cells, $\mathrm{T}$ follicular regulatory (Tfr) cells were first identified in mice as simultaneously expressing $\mathrm{Tfh}$ cell-associated molecules (such as CXCR5, ICOS, PD-1, and the transcription factor Bcl6) and Treg cell-characterized proteins (represented by Foxp3) ${ }^{14-16}$. Although sharing nearly identical receptors with Tfh cells, Tfr cells, which are derived from Treg cells exert a potent inhibitory effect that suppress excessive Tfh cell expansion, B cell response and antibody production to orchestrate germinal center (GC) cell dynamics ${ }^{17-19}$. Growing evidence has shown that the dysregulations of Tfr cells including quantity and quality anomalies, as well as the imbalance of Tfh/Tfr cells, are implicated in various immune diseases, such as autoimmunity, chronic inflammatory disease, malignancy, and transplant rejection ${ }^{19-28}$.

However, the role of Tfr cells during pregnancy still remains unelucidated. Here, we introduced an allogeneic normal pregnant mouse model and found that CD $4^{+}$CXCR $5{ }^{\text {hi }}$ PD- $1^{\text {hi }}$ Foxp $3^{+}$Tfr cells were preferentially accumulated in the uterus at mid-gestation and displayed a distinct phenotype. In addition, the absence of PDL1 resulted in increased fetal resorption by favoring Tfr cells accumulation and upregulating PD-1 expression on these cells. However, PDL1 blockage altered neither the ratio of $\mathrm{Tfh} / \mathrm{Tfr}$ cells nor B-cell maturation and differentiation. Collectively, our findings present a correlation of Tfr cells accumulation with healthy allogeneic pregnancy and PDL1 blockade-induced miscarriage, and suggest that Tfr cells may be engaged in the maintenance of maternalfetal immune tolerance. Furthermore, although blockade of PD-1/PD-L1 checkpoint has been proven to be an effective immunotherapy in some tumors ${ }^{29-31}$, the reproductive safety must be considered because of the increased Tfr cells and fetal losses in PDL1 blocked pregnant mice.

\section{Materials and methods \\ Mice}

Pathogen-free female BALB/c $\left(\mathrm{H}-2^{\mathrm{d}}\right)$ and male C57BL/6 $\left(\mathrm{H}-2^{\mathrm{b}}\right)$ mice (8-10-week-old) were purchased from the Shanghai Laboratory Animal Center, Chinese Academy of Science, and bred in the Department of Laboratory Animal Science, Shanghai Jiaotong University School of Medicine (Shanghai, China). Two adult BALB/c females were co-caged with one C57BL/6 male, and the presence of a vaginal-plug was denoted as embryonic day 0.5 (E0.5). All animal experiments were ethically acceptable and performed in accordance with guidelines for animal care and use in research of Shanghai Jiaotong University School of Medicine (Shanghai, China).

\section{Preparation of mononuclear cells}

Pregnant mice were sacrificed either on E11.5 or E18.5. Isolation and incubation of mononuclear cells from peripheral blood, spleen, thymus, uterus, and bone marrow was conducted as previously described ${ }^{13,32,33}$. In brief, whole blood was harvested from mice via vena orbitalis into phosphate buffered saline (PBS) containing 2\% EDTA after intraperitoneal anesthesia with pentobarbital sodium at a dosage of $50 \mathrm{mg} / \mathrm{kg}$. Blood cell suspension was layered by using Lymphoprep ${ }^{\text {TM }}$ (AS1114546, Axis-shield) and peripheral blood mononuclear cells (PBMCs) were isolated according to a standard density gradient centrifugation process. The collection of spleen and thymus cells required a syringeplunger to grind the tissue gently and thoroughly, and a $70-\mu \mathrm{m}$ pore size cell strainer (352350, BD Biosciences) for cell suspensions filtering through. Purified splenic and thymic mononuclear cells were gathered by centrifugation and counted by using a Leica DMI 3000B microscope with trypan blue exclusion after potential contaminating red blood cells being wiped out under the influence of co-incubation with ACK Lysing Buffer (A10492-01, Gibco) for $1 \mathrm{~min}$. To obtain single mononuclear cell suspensions in the uterus, embryos were removed carefully after hysterolaparotomy. The pooled uterus and placentas were finely cut into less than $1 \times 1 \times$ $1 \mathrm{~mm}$ pieces with ocular scissors, and the minced tissue was then dispersed in PBS and filtered through a $70-\mu \mathrm{m}$ pore size cell strainer, followed by being purified through density gradient centrifugation with Lymphoprep ${ }^{\mathrm{TM}}$. Mice femurs were introduced to isolate mononuclear cells derived from bone marrow, followed by centrifugation to harvest mononuclear cell suspensions.

\section{Treatment protocol}

To induce embryo loss, $250 \mu \mathrm{g}$ anti-mouse PDL1 blocking monoclonal antibody (mAb) (clone: 10 F.9G2, 
BioLegend) was administered to each pregnant mouse in experimental group via intraperitoneal (i.p.) injection on E5.5 and E8.5, consecutively. As controls, mice were i.p. treated with the equivalent dosage of IgG at the same gestation day. Both IgG-treated controls and PDL1blocked pregnant mice were sacrificed on E11.5, and the embryo resorption rate was calculated as: Resorption rate $(\%)=[$ number of resorbed embryos/number of total (resorbed + viable) embryos] $\times 100$.

\section{mAbs and reagents}

Fluorescein-conjugated anti-mouse mAbs, including anti-CD4-Pacific Blue (clone: RM4-5), anti-CD8-V500 (53-6.7), anti-CXCR5-PE-Cy7 (2G8), anti-CD95 (FAS)PE-Cy7 (Jo2), and anti-CD138-APC (281-2) were purchased from BD Pharmingen; anti-FOXP3-PE-Cyanine 5 (FJK-16s) and anti-GL7-Alexa Fluor 488 (GL-7) were from eBioscience; and anti-CD19-PE (6D5), anti-CD279 (PD-1)-PE (RMP1-30), anti-BCL-6-APC (7D1), and antiIgG (minimal x-reactivity)-PerCP-Cy5.5 (Poly4053) were from BioLegend.

\section{Flow cytometry}

Surface and intranuclear stainings were carried out by multicolor flow cytometry (FCM) as we and others described previously ${ }^{34-37}$. For cell surface staining, freshly isolated mononuclear cells were resuspended in $100 \mu \mathrm{L}$ PBS containing 3\% (v/v) fetal bovine serum (FBS) with different fluorescein-conjugated mAbs. After incubation at room temperature in the dark for $30 \mathrm{~min}$, the labeled cells were washed with PBS twice and collected for later use. Intranuclear staining of Foxp3 and BCL- 6 was performed in line with the instruction for Foxp3 staining (00-5523-00, eBioscience) after surface combination. Cells were fixed and permeabilized using Fixation/Permeabilization Buffer for $1 \mathrm{~h}$, followed by being incubated with directly conjugated specific antibody for $40 \mathrm{~min}$ at room temperature. Finally, we used a BD FACS Canto II flow cytometer (BD Biosciences, USA) to collect the immunostained cells and the FlowJo 7.6.1 software to analyze the data.

\section{Statistical analyses}

Statistical analyses were implemented in Graphpad Prism 5 software and the results were shown as mean \pm standard error of means (M. \pm S.E.M.). The normality of our data was evaluated by the Shapiro-Wilk normality test. If the data followed normal distribution, we performed statistical analysis with unpaired or paired Student's $t$-test, or one-way ANOVA with subsequent Tukey's post-tests; if not, we analyzed the data by using the Mann-Whitney $U$ or Wilcoxon matched pairs test, or Kruskal-Wallis test followed by Dunns multiplecomparison test. $P$-values $<0.05$ were considered to be significant difference. Details are described in the Figure Legends. Randomization, blinding, and sample size estimation tests were not done for our animal studies.

\section{Results}

Result 1: CD4 ${ }^{+}$CXCR5 ${ }^{\text {hi PD- }}{ }^{\text {hi }}$ Foxp $3^{+}$Tfr cells are preferentially accumulated in the uterus at mid-gestation

Tfr cells have been usually defined as the CXCR ${ }^{\text {hi }}$ PD$1^{\text {hi }}$ Foxp $^{+}$population gated in $\mathrm{CD}^{+}{ }^{+} \mathrm{T}$ cells ${ }^{19,22}$. To identify whether $\mathrm{Tfr}$ cells are involved in immune homeostasis at the maternal-fetal interface during allogeneic-normal pregnancy, adult BALB/c females were mated with C57BL/6 males and the presence of a vaginalplug was taken for embryonic day 0.5 (E0.5). We performed multi-color flow cytometry (FCM) to screen CD $4^{+}$CXCR $5{ }^{\text {hi }}$ PD $-1^{\text {hi }}$ Foxp $3^{+}$Tfr cells among different lymphoid tissues, and found that the proportion of these cells was remarkably higher in the uterus than that in the peripheral blood $(\mathrm{PB})$, spleen and thymus on E11.5 (Fig. 1a, c). Moreover, the proportion of $\mathrm{CD} 4^{+} \mathrm{CXCR} 5^{\text {hi }}$ PD- ${ }^{\text {hi }}{ }^{\text {Foxp }} 3^{+}$Tfr cells in the uterus was significantly reduced on E18.5 than E11.5 (Fig. 1b, c), but very few CD $4^{+}$CXCR $5{ }^{\text {hi PD }-1}{ }^{\text {hi }}$ Foxp $3^{+}$Tfr cells were detected in the uterus on E5.5-7.5 (Supplementary Fig. 1). However, although the absolute number of splenic $\mathrm{CD} 4^{+} \mathrm{CXCR} 5^{\text {hi }}$ PD- $1^{\text {hi }}$ Foxp $3^{+}$Tfr cells dramatically increased on E11.5 and then returned to the normal level on E18.5, the proportion of these cells was not changed in the spleen during pregnancy (Supplementary Fig. 2). Taken together, these data indicate that $\mathrm{CD} 4^{+} \mathrm{CXCR}^{\mathrm{hi}} \mathrm{PD}-\mathrm{1}^{\mathrm{hi}} \mathrm{Foxp} 3^{+} \mathrm{Tfr}$ cells are dominantly enriched in the uterus at midgestation.

\section{Result 2: uterine Tfr cells display a distinct phenotype}

As previous studies have demonstrated, Tfr cells not only express similar surface antigens and transcription factors to Tfh cells including CXCR5, PD-1, ICOS, and BCL-6, but also are characterized by typical Treg cell markers such as Foxp3, and Tfr and Tfh cells can be distinguished based on Foxp3 expression ${ }^{14,19}$. Similarly, we identified Tfr cells as CXCR5 $5^{\text {hi }} \mathrm{PD}-1^{\text {hi }}$ Foxp $3^{+}$population and Tfh cells as CXCR5 $5^{\text {hi }} \mathrm{PD}-1^{\text {hi }}$ Foxp $3^{-}$population, both of which were gated in $\mathrm{CD} 4^{+} \mathrm{T}$ cells (Fig. 2a).

To characterize the Tfr cells residing at the uterus, FCM was implemented to determine the expression of characteristic proteins at mid-gestation. Surprisingly, the level of BCL-6, PD-1 and CXCR5 was notably higher in uterine Tfr than Tfh cells (Fig. 2b-d). In addition, uterine Tfr cells presented a higher expression of PD-1 as compared with that in the $\mathrm{PB}$ and spleen, as well as a greater frequency of CXCR5 than that in the spleen and thymus (Fig. 2e-h). However, Tfr cells residing in the uterus and other lymphoid tissues displayed no palpable difference in the expression of BCL-6 and Foxp3 


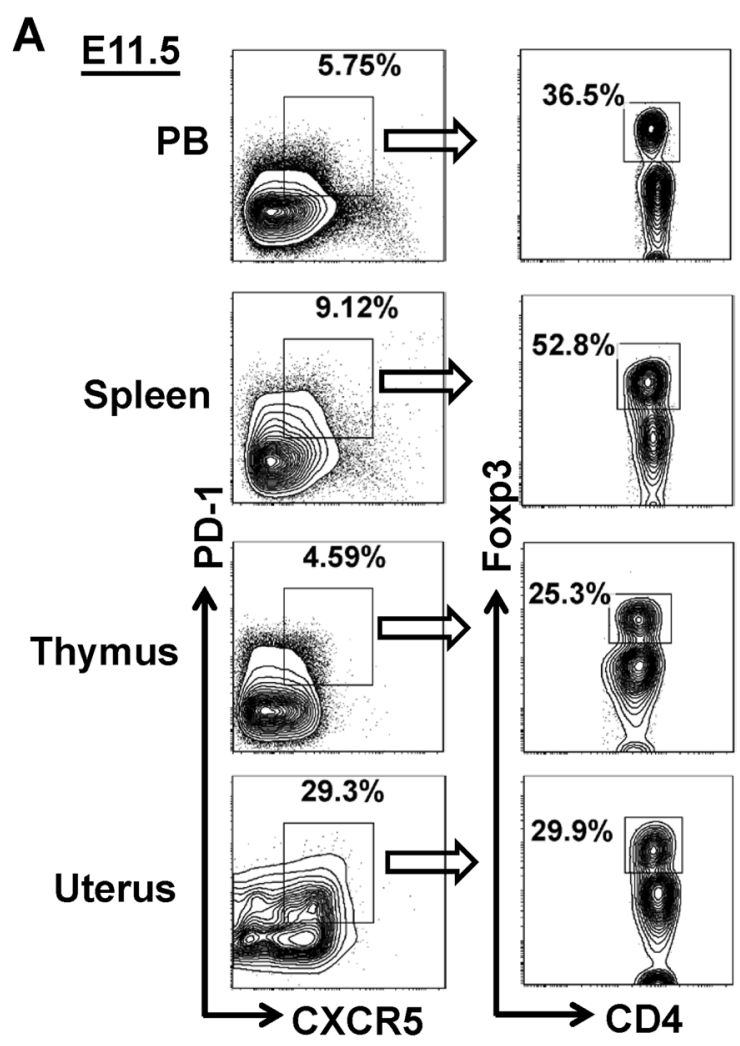

B
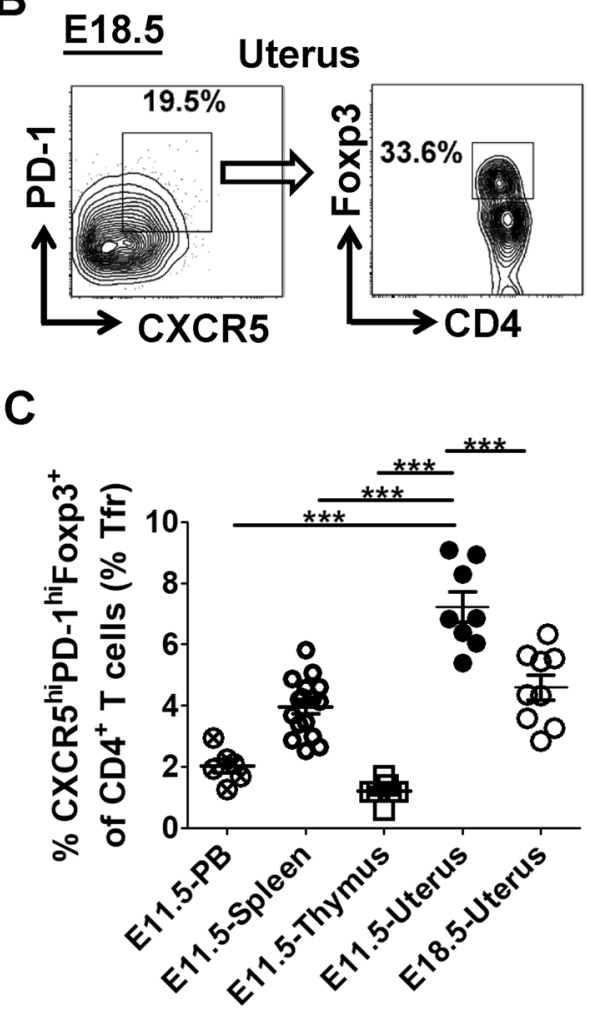

Fig. $1 \mathrm{CD}^{+}{ }^{+}$CXCR5 $^{\text {hi }}{ }^{\text {PD- }} 1^{\text {hi }}$ Foxp $^{+}{ }^{+}$Tfr cells are preferentially accumulated in the uterus at mid-gestation. Adult female BALB/C and male C57BL/6 mice were co-caged in a 2:1 ratio and the presence of a vaginal-plug was taken for embryonic day 0.5 (E0.5). a-c Representative flow cytometric plots $(\mathbf{a}, \mathbf{b})$ and cumulative data (c) illustrating the percentage of CXCR5 hi PD- $1^{\text {hi }}$ Foxp3 ${ }^{+}$population gated in CD4 ${ }^{+}$T cells derived from the $\mathrm{PB}$, spleen, thymus and uterus of maternal mice on E11.5 $(\mathbf{a}, \mathbf{c})$ as well as from the uterus on E18.5 $(\mathbf{b}, \mathbf{c})$. Each symbol reflects the data from a single mouse ( $n \geq 7$ mice per group) and the data are representative of two independent experiments. The cells are gated in CD4 ${ }^{+} T$ cells. Data were assessed statistically using one-way ANOVA followed by Tukey's multiple-comparison test. PB peripheral blood; hi high; ***p $<0.001$.

(Supplementary Fig. 3). These results verify that uterine Tfr cells display a distinct phenotype as compared with those in other lymphoid tissues as well as uterine Tfh cells.

\section{Result 3: PDL1 blockage increases fetal resorption and Tfr cells}

To induce an abortion mouse model, pregnant BALB/c females, which had been mated with $\mathrm{C} 57 \mathrm{BL} / 6$ males, were administrated intraperitoneally with anti-PDL1 blocking monoclonal antibody (mAb) or IgG (as a nonspecific control) at a dosage of $250 \mu \mathrm{g}$ on E5.5 and E8.5, respectively. Mice with both treatments were sacrificed on E11.5. The whole uterus was separated and more embryo losses were visibly observed in the PDL1-blocked group (Fig. 3a, b), which was consistent with previous reports $^{12,13}$. Furthermore, the proportion as well as the absolute number of $\mathrm{CD} 4^{+}$CXCR $5{ }^{\text {hi }}$ PD $-1^{\text {hi }}$ Foxp $3^{+}$Tfr cells in the spleen, thymus, and uterus exhibited an apparent increase after the administration of anti-PDL1
mAb (Fig. 3c-h and data not shown), suggesting that the higher rate of fetal resorption due to PDL1 blockade may be associated with the enhanced accumulation of Tfr cells.

\section{Result 4: PDL1 blockage upregulates PD-1 expression on Tfr cells}

Next, we explored whether the expression of characteristic molecules on Tfr cells was also altered after treating pregnant mice with anti-PDL1 mAb. Our results demonstrated that the expression of PD-1 on CD $4^{+}$CXCR $5{ }^{\text {hi }}$ PD $-1^{\text {hi }}$ Foxp $3^{+}$Tfr cells from PDL1-blocked mice was dramatically higher than that from the controls in the uterus, spleen, $\mathrm{PB}$, and thymus, while the expression of other characteristic proteins including CXCR5, BCL-6, and Foxp3 on/in Tfr cells displayed no significant difference between anti-PDL1treatment and control groups in the uterus and other lymphoid tissues (Fig. 4 and Supplementary Fig. 4). These data suggest that the increased fetal resorption resulted from PDL1 absence may correlate with the high expression of PD-1 on Tfr cells. 


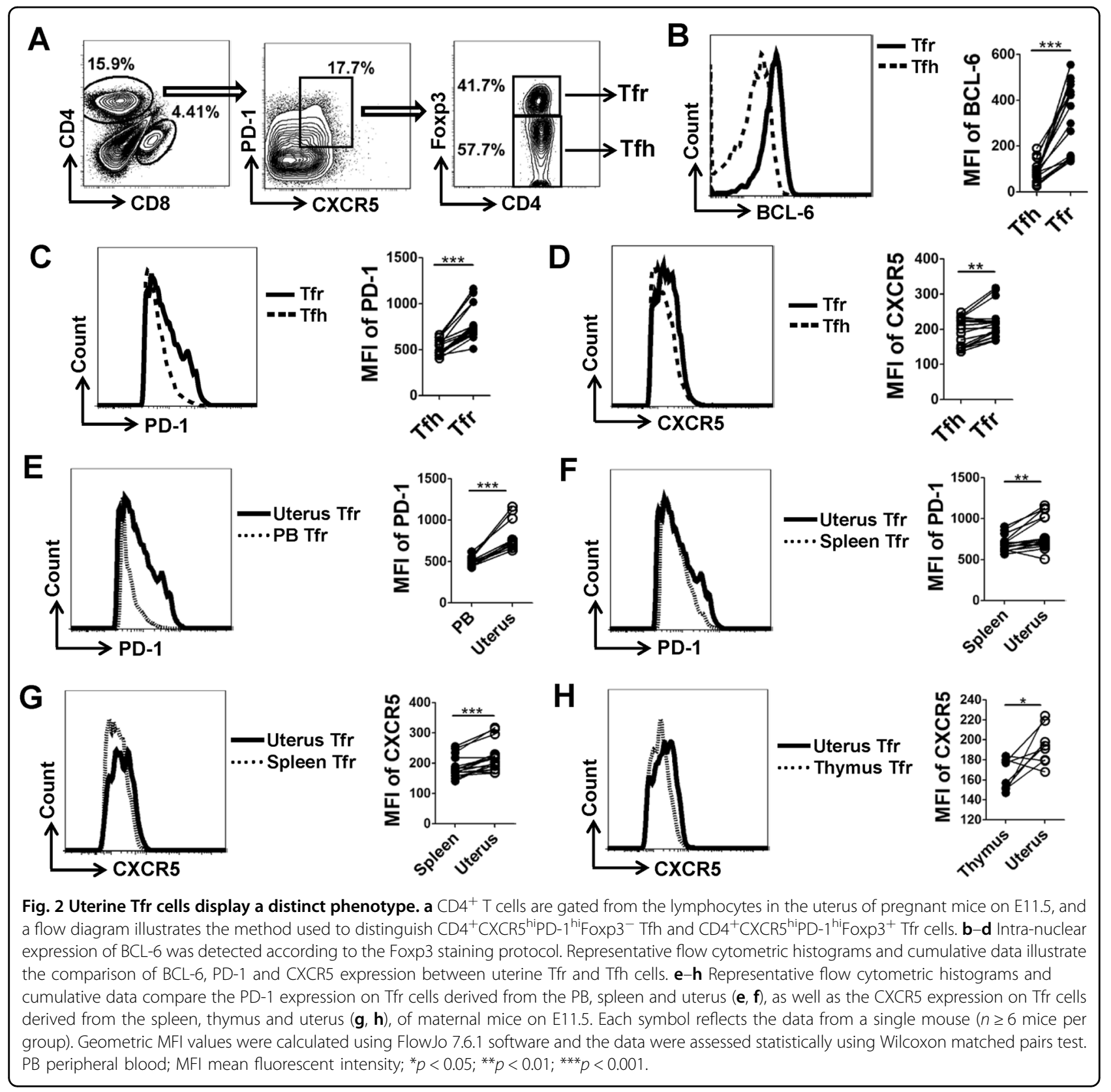

Result 5: PDL1 blockage does not affect the ratio of Tfh/Tfr cells

Tfh cells are essential for GC formation and maintenance, and provide survival signals for high affinity $\mathrm{B}$ cells within the GC for their differentiation to isotypeswitched antibody secreting cells and memory B cells ${ }^{38,39}$. Although the function of Tfr cells in the GC has not been completely clarified, the mainstream view currently is that Tfr cells restrict Tfh- and B-cell proliferation and exert an inhibitory action on antibody production ${ }^{40-42}$. The ratio of Tfh/Tfr cells plays a crucial role in humoral immunity and regulates antibody responses, and effective humoral immunity depends on the delicate balance between promotive Tfh cells and suppressive $\mathrm{Tfr}$ cells ${ }^{28,43}$. Our previous study ${ }^{13}$ and above data (Fig. 3) showed that PDL1 blockage increased both Tfh and Tfr cells simultaneously. Hence, the ratio of $\mathrm{Tfh} / \mathrm{Tfr}$ cells was further examined, and we found that the ratio showed no statistical difference between PDL1-blocked and control mice, accompanied by no difference in the proportion of Foxp $3^{+}$cells among $\mathrm{CD} 4^{+} \mathrm{CXCR} 55^{\mathrm{hi}} \mathrm{PD}-1^{\mathrm{hi}}$ population between these mice (Fig. 5). 


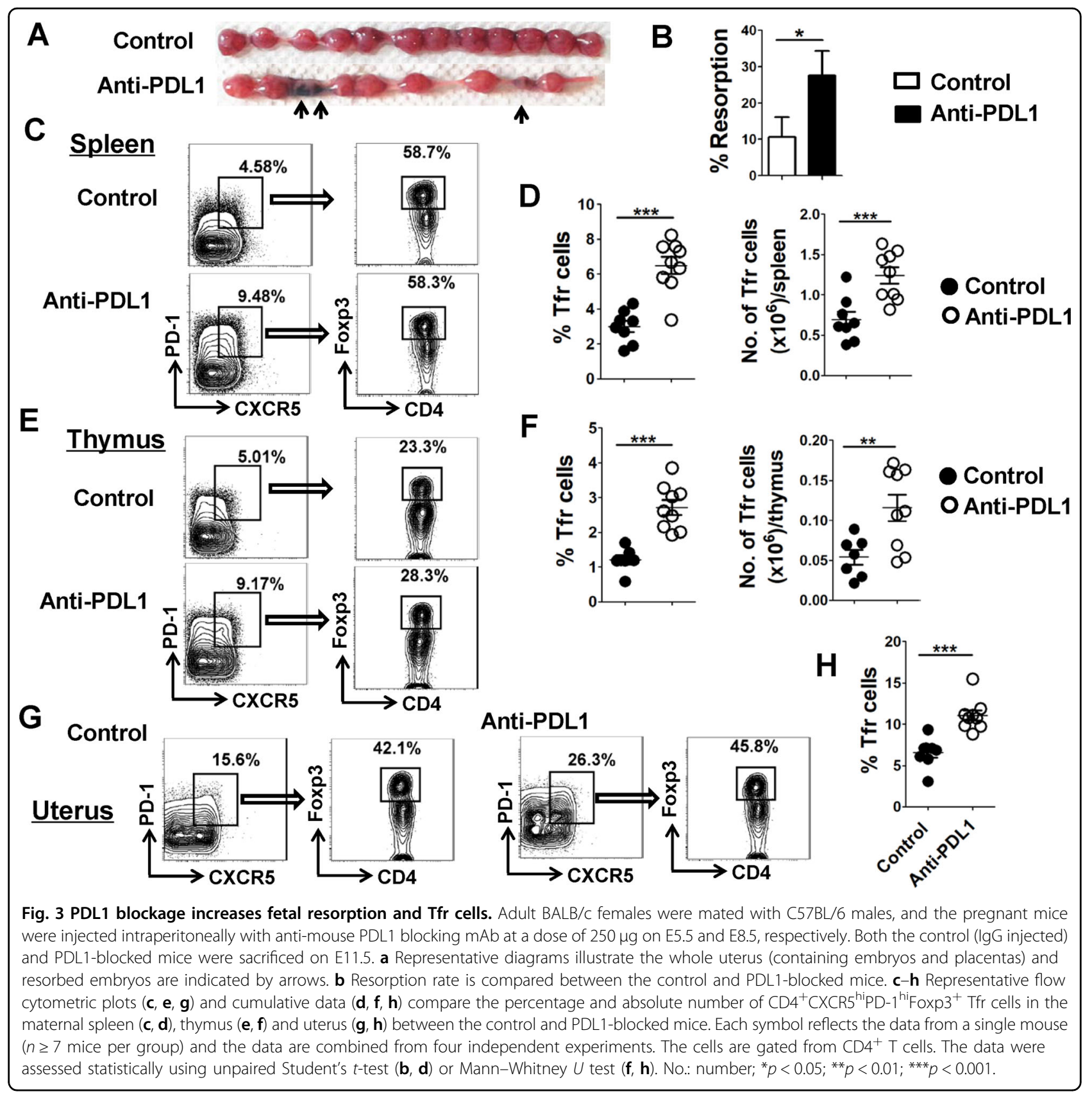

Result 6: PDL1 blockage does not affect B-cell maturation and differentiation

Given the inhibitory effect of Tfr cells, finally, we asked whether PDL1 blockade impact B-cell maturation and differentiation. However, the proportion of $\mathrm{CD} 19^{+}$total $\mathrm{B}$ cells; the expression of GC-resident $\mathrm{B}$ cell markers including CD138, GL7, FAS, and IgG; together with the proportion of $\mathrm{CD} 138^{+}$plasma cells, $\mathrm{IgG}^{+}$antibodyproducing B cells and $\mathrm{GL}^{+} \mathrm{FAS}^{+} \mathrm{GC}$ B cells; were not apparently different between the control and PDL1blocked mice in the bone marrow (BM), spleen, PB and uterus (Figs. 6 and 7 and Supplementary Figs. 5 and 6).
Above all, there is a paucity of convincing evidence concerning the role of PDL1 blockade in the maturation and differentiation of $\mathrm{B}$ cells.

\section{Discussion}

Multiple mechanisms, the redistribution of immune cells in particular, contribute to the elaborate balance of immune clearance and immune tolerance at the maternalfetal interface. Putative CD $4^{+}$effector T helper (Th) cells Th1/Th2/Th17 and Treg paradigms gave their importance in fetal graft rejection and adoption, and either bias may result in different kinds of pregnancy failures including 
A
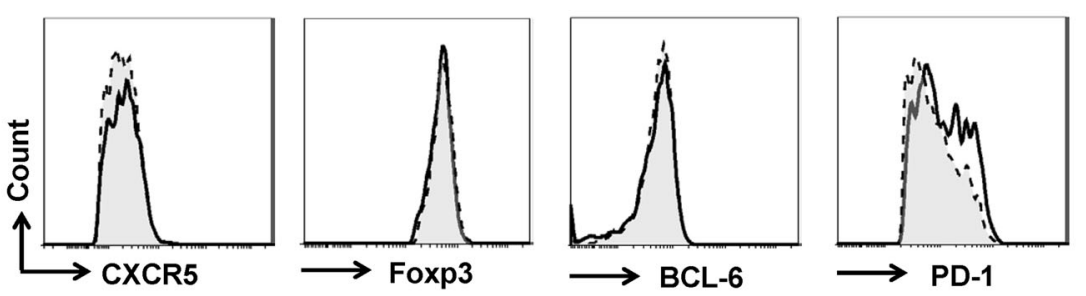

B
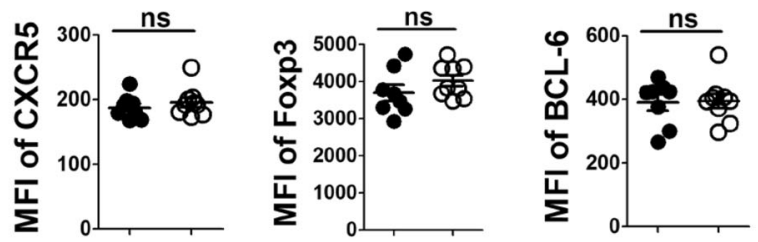

C
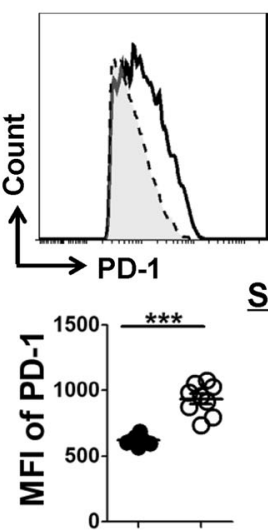

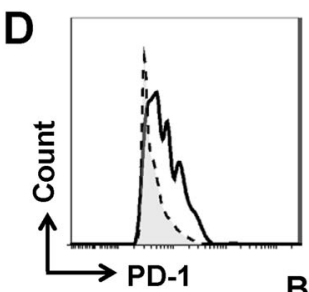

Spleen

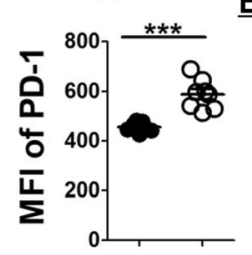

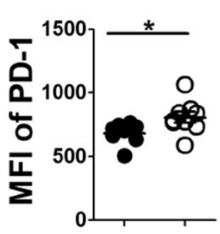

E

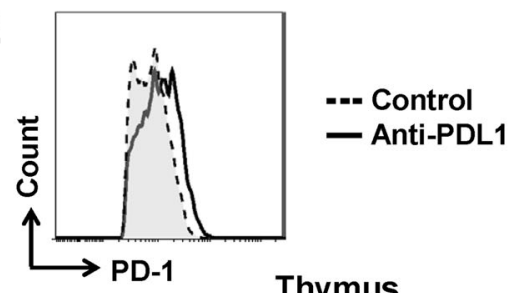

Thymus

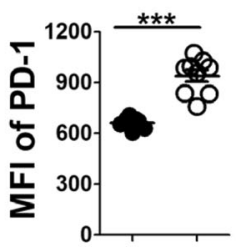

$\underline{\text { Uterus }}$

- Control

O Anti-PDL1

-- Control

- Anti-PDL1

Thyms

Control

O Anti-PDL1

Fig. 4 PDL1 blockage upregulates PD-1 expression on Tfr cells. $\mathbf{a}$, $\mathbf{b}$ Representative flow cytometric histograms and cumulative data compare the molecular expression of CXCR5, Foxp3, BCL-6 and PD-1 on/in uterine Tfr cells between the control and PDL1-blocked mice. c-e Representative flow cytometric histograms and cumulative data illustrating the expression level of PD-1 on Tfr cells derived from the spleen (c), blood (d), and thymus (e) of the control and PDL1-blocked mice. Each symbol reflects the data from a single mouse ( $n \geq 5$ mice per group). Cells are gated in CD4 ${ }^{+}$CXCR5 ${ }^{\text {hi }}$ PD- $1^{\text {hi }}$ Foxp $3^{+}$Tfr cells. Geometric MFI values were calculated using FlowJo 7.6 .1 software and the data were assessed statistically using unpaired Student's $t$-test. MFI mean fluorescent intensity; ns not significant; ${ }^{*} p<0.05$; ${ }^{* * *} p<0.001$.

spontaneous abortion, preterm delivery, pre-eclampsia, fetal growth restriction, and even death ${ }^{44-46}$.

The newly concerned Tfr cells have attracted much interest due to their unique roles in immunoregulation. Previous studies have demonstrated that this subpopulation of $\mathrm{CD}^{+} \mathrm{T}$ cells co-opts $\mathrm{Tfh}$ differentiation pathway and upregulates the transcriptional repressor $\mathrm{Bcl}-6$ which is required for the generation of $\mathrm{Tfh}$ cells and the suppression of Th1, Th2, and Th17 cell differentiation ${ }^{15,47,48}$. Recently, we proposed Tfh cells as a crucial player involved in the gestation, enriching the original paradigm into a novel Th1/ Th2/Th17/Treg/Tfh paradigm ${ }^{13}$. Our data showed that Tfh cells were clustered in the uterus at mid-gestation, however, PDL1-blockade-induced excessive abundance of Th cells might step back the development and lead to embryo loss ${ }^{13}$. Since Tfh and Tfr cells share a similar phenotype but an opposite function, we next investigated the characteristics and roles of Tfr cells during pregnancy.
Herein, we defined Tfr cells as CXCR $5^{\text {hi } P D-1} 1^{\text {hi }}$ Foxp $3^{+}$ population gated in $\mathrm{CD}^{+} \mathrm{T}$ cells as previous studies performed ${ }^{19,22}$. Interestingly, we found that CD ${ }^{+}{ }^{\text {CXCR }} 5^{\text {hi }}$ PD $-1^{\text {hi }}$ Foxp $3^{+}$Tfr cells were more prone to enriched in the uterus at mid-gestation (Fig. 1), and the proportion of splenic CD $4^{+}$CXCR $5^{\text {hi }}$ PD- $1^{\text {hi }}$ Foxp3 ${ }^{+}$Tfr cells was not significantly changed after gestation (Supplementary Fig. 2). These data confirmed a redistribution of Tfr cells during normal pregnancy. Furthermore, human decidual tissues and peripheral blood samples were collected from healthy women who were undergoing early elective abortions in the first trimester of pregnancy (at 6-12 weeks of gestation), and flow cytometry analysis showed that the proportion of $\mathrm{CD} 4{ }^{+} \mathrm{CXCR} 5{ }^{\text {hi }} \mathrm{PD}$ $1^{\text {hi }}$ Foxp $3^{+} \mathrm{Tfr}$ cells was significantly higher in human deciduas than that in peripheral blood (Supplementary Fig. 7). These data were consistent with our observation in the allogeneic pregnancy mouse model (Fig. 1). 
A

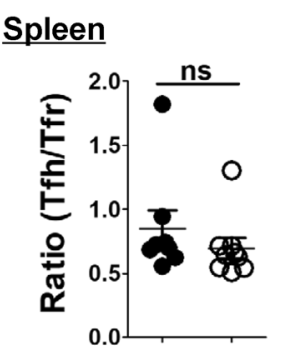

B

Spleen

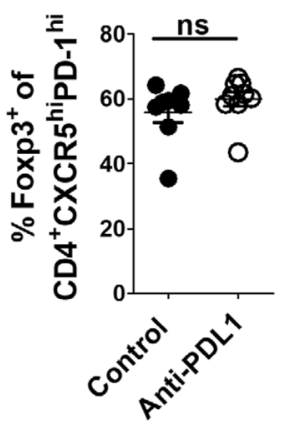

Thymus

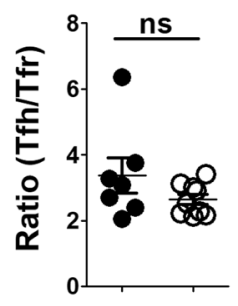

Thymus

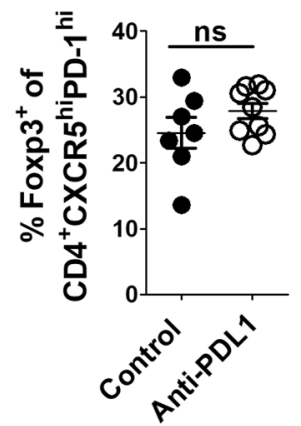

$\underline{\text { Uterus }}$

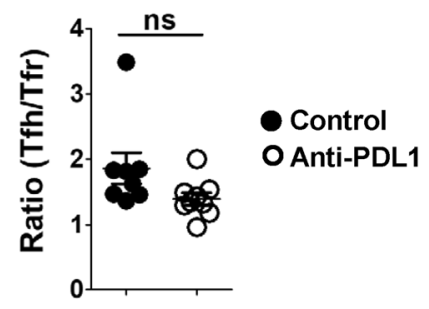

$\underline{\text { Uterus }}$

Fig. 5 PDL1 blockage does not affect the ratio of Tfh/Tfr cells. $\mathbf{a}, \mathbf{b}$ Cumulative data comparing the ratio of Tfh/Tfr cells (a) as well as the percentage of Foxp $3^{+}$population among CD4 ${ }^{+}$CXCR5 ${ }^{\text {hipD-1 }} 1^{\text {hi }} T$ cells $(\mathbf{b})$ derived from the spleen, thymus, and uterus between the control and PDL1blocked mice on E11.5. Each symbol reflects the data from a single mouse ( $n \geq 7$ mice per group) and the data are combined from four independent experiments. The data were assessed statistically using Mann-Whitney $U$ test. ns not significant.

An earlier RNA-seq data demonstrated that Tfr cells showed a peculiarly higher expression of CXCR5, and another publication revealed that Tfr cells displayed a greater frequency of PD-1 than $\mathrm{Tfh}$ cells ${ }^{49,50}$. Almost coherently, we found that the level of BCL-6, PD-1 and CXCR5 was notably higher in uterine Tfr than Tfh cells (Fig. 2a-d). Previous studies have shown that PDL1 is highly expressed on the fetal extravillous trophoblast cells (EVTs) and decidual stroma cells (DSCs) during human and mouse early pregnancies ${ }^{4,51,52}$, and DSCs can secrete C-X-C motif chemokine 13 (CXCL13), which may contribute to a high level of this chemokine detected in the amniotic fluid of pregnant women ${ }^{53,54}$. Therefore, we speculate that DSCs/EVTs-derived PDL1 (a prior binding ligand of PD-1) and CXCL13 (the only known ligand for CXCR5) facilitate the recruitment and retention of Tfr cells with a high expression of PD-1 and CXCR5 in the uterus, which contribute to our observation that uterine Tfr cells display a distinct phenotype as compared with those in other lymphoid tissues (Fig. 2e-h).

Despite antibody-mediated rejection (AMR) is dominantly attributed to $\mathrm{B}$ cells maturation and specific antibody production, $\mathrm{T}$ cells are considered as principle infiltration cells in the respect of rejection ${ }^{55}$. Tfr cells are believed to induce the inability of $\mathrm{Tfh}$ cells in the GC, specifically preventing incessant antibody-produced B cell responses. Subsequent evidence suggested that Tfr cells effectively regulated humoral immune responses in autoimmune diseases and organ transplantation ${ }^{19,56-59}$. A leading theory is that Tfr cells are formidable adversaries against chronic graft-versus-host diseases (cGVHD) ${ }^{59,60}$. In patients with chronic renal allograft rejection, the number and the ratio of Tfr cells were significantly attenuated compared with non-AMR patients, while IL-21producing Tfh cells were obviously augmented, indicating that Tfr cells can remove barriers to facilitate a successful transplant ${ }^{58}$. The semi-allogeneic fetus is long regarded as a natural transplantation model in vivo, that allograft rejection may lead to miscarriage or other pregnancy complications ${ }^{61}$. Consistent with the mainstream findings in other transplantations ${ }^{58,62,63}$, our results showed a higher proportion of $\mathrm{CD} 4{ }^{+} \mathrm{CXCR} 5^{\mathrm{hi}} \mathrm{PD}-1^{\mathrm{hi}} \mathrm{Foxp} 3^{+} \mathrm{Tfr}$ cells infiltrated in the uterus (Fig. 1), indicating that Tfr cells may participate in maintaining the harmony between the allogeneic fetus and the matrix.

PD-1 is a proverbial inhibitory receptor expressed by $T$ cells, B cells, monocytes, and dendritic cells (DCs), and B cells and DCs express its ligands, PDL1 and PDL2 ${ }^{64}$. The PD-1-PDL1 signaling pathway has been widely regarded as a major contributor in maintaining 


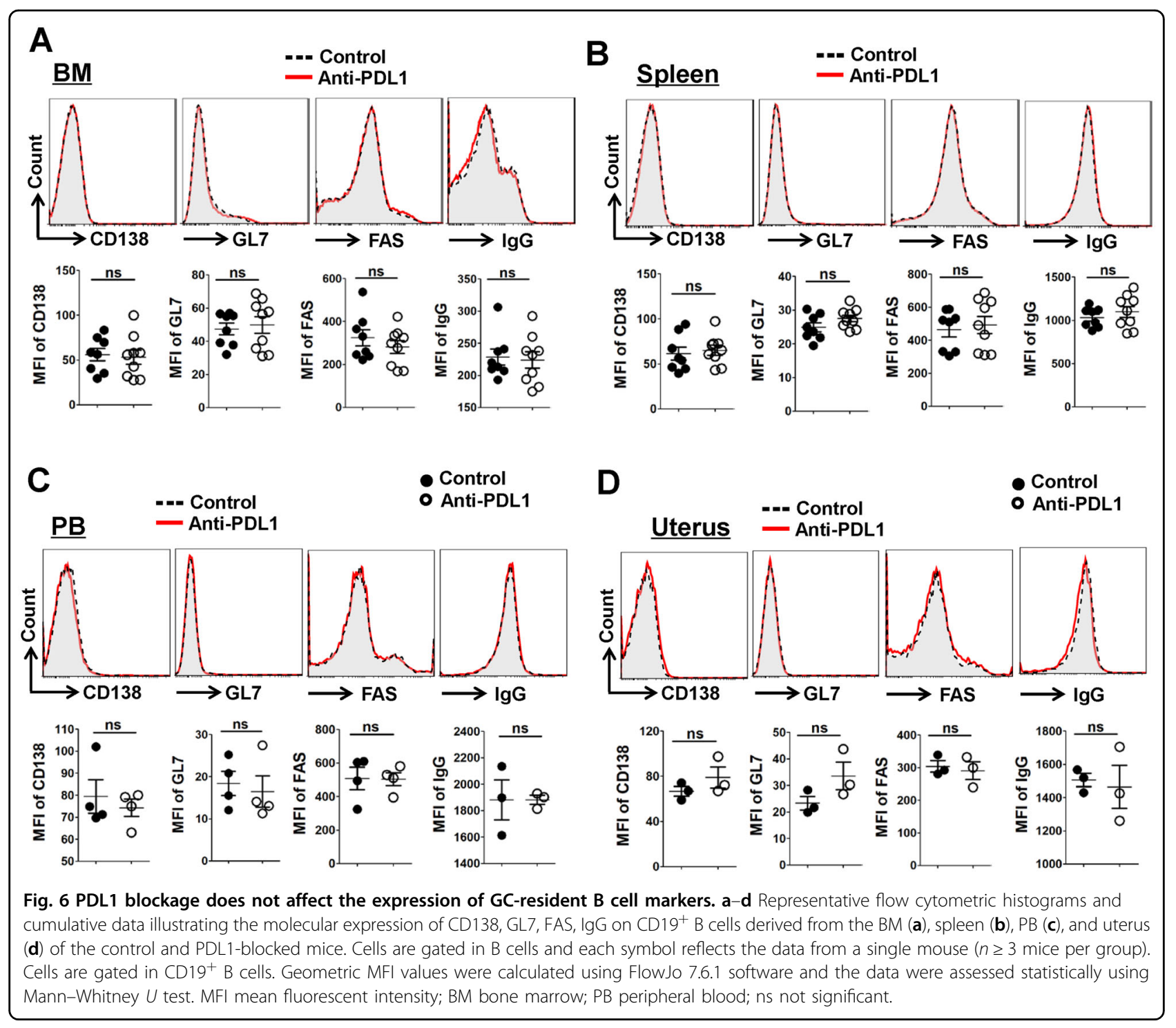

fetomaternal immune tolerance. First, Indira Guleria, et al. found that blockade of PDL1 signaling resulted in increased fetal rejection during murine allognenic pregnancy but not syngeneic pregnancy, and this rejection depended on $\mathrm{T}$ cells but not B cells and APCs by using RAG-1-deficient and B cell-deficient mice ${ }^{52}$. Subsequently, they demonstrated that PDL1 blockade led to an expansion of both Th1 and Th17 cells but a suppression of Treg and Th2 cells ${ }^{10,12,52}$. Recently, we proposed Tfh cells as a key player involved in the gestation and found that the increased foetal resorption by PDL1 blockade was accompanied by enhanced accumulation of Tfh cells ${ }^{13}$. In this study, our data showed that PDL1 blockage increased CD $4^{+}$CXCR $5{ }^{\text {hi }}$ PD $-1{ }^{\text {hi }}$ Foxp $3^{+}$Tfr cells but did not affect B-cell maturation and differentiation, and our study is the first report using an allognenic model pregnancy to establish a link among PD-1-PDL1 signaling pathway, Tfr cells, and fetomaternal immune tolerance. Therefore, our data together with previous studies indicated that PD-1-PDL1 signaling pathway plays a key role in maintaining fetomaternal tolerance by favoring the expansion of Th1, Th17, Tfh and Tfr cells but limiting the expansion of Th2 and Treg cells.

Interestingly, PD-1 expression on Tfr cells was observed to be significantly upregulated in PDL1-deficient mice (Fig. 4), and we proposed two possible explanations for this finding. (1) Although the role of PD-1/PDL1 pathway in $\mathrm{T}$ cell exhaustion has attracted a great deal of attention, PD-1 is not considered as an exhaustion-specific mar$\mathrm{ker}^{5,65}$. PD-1 is also highly expressed by all $\mathrm{T}$ cells during activation as a natural brake to fine-tune the $\mathrm{T}$ cell responses, which is an important regulatory manner in normal host physiology ${ }^{5}$. In this study, we used anti-PDL1 blocking antibody to disturb the interaction between PD- 


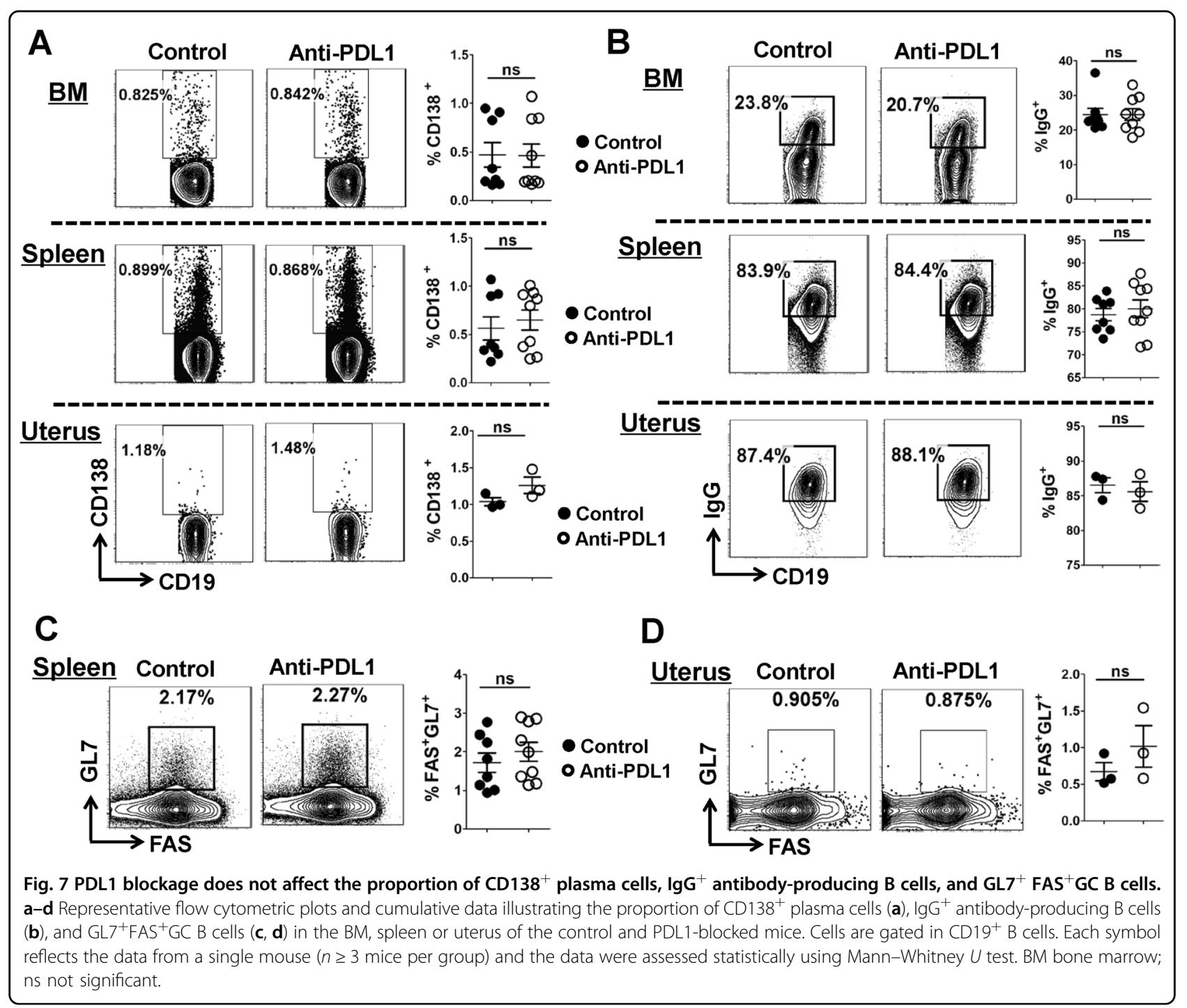

1 and PDL1. The PD-1 signaling in Tfr cells was interfered for lacking engagement of the ligand PDL1, which might result in the activation of Tfr cells accompanied by the upregulated expression of PD-1 on these cells. (2) Critical lack of PD-1/PDL1 interaction by blocking PDL1 promoted Tfr cells to express more PD-1 to seek for more effective combination between PD-1 and PDL1, and to receive more signals provided by the ligand PDL1. Overall, several reports have shown that PDL1 blockade leads to an increase in the number and function of $\mathrm{Tfr}$ cells ${ }^{13,20,50}$. However, we hold the opinion that the surface receptor PD-1 cannot deliver inhibitory signals without following its engagement with PDL1, and it is the attenuation of PD-1 signaling rather than the augmented expression of PD-1 that contribute to the greater abundance and enhanced capability of Tfr cells.

Successful humoral immunity as well as B cell immunity depends on the intricate balance between stimulatory $\mathrm{Tfh}$ cells and inhibitory $\mathrm{Tfr}$ cells ${ }^{18}$. Sage et al. revealed that the PD-1/PDL1 pathway controls not only the generation of Tfr cells but also the ratio of Tfh to Tfr cells in the lymph nodes and blood ${ }^{50}$. As Tfr cells exert an inhibitory role in restricting the over-abundance of Tfh and $\mathrm{B}$ cells, as well as suppressing the antibody-producing $B$ cell responses in the $\mathrm{GC}^{40-42}$, we wondered if PDL1 blockade-related Tfr cells accumulation had an impact on Tfh/Tfr cells ratio and B cells maturation. Surprisingly, we found that neither the ratio of $\mathrm{Tfh} / \mathrm{Tfr}$ cells nor the proportion of Foxp $3^{+}$cells among $\mathrm{CD} 4^{+}$CXCR5h ${ }^{\mathrm{i}} \mathrm{PD}-1^{\text {hi }}$ population in the spleen, thymus and uterus was altered in a statistically significant manner (Fig. 5). Beyond that, PDL1 deficiency appeared no impact on B-cell maturation and differentiation in the BM, spleen, PB or uterus (Figs. 6 and 7 and Supplementary Figs. 5 and 6).

Consistent with our findings that PDL1 blockage increases both $\mathrm{Tfh}^{13}$ and Tfr cells (Fig. 3), growing 
evidence has shown that the deficiency of PD-1 or PDL1 results in more Tfh cells and/or Tfr cells ${ }^{20,50,66}$. However, some studies have shown that the humoral responses are enhanced ${ }^{67-69}$ while others found that they are weakened ${ }^{70-72}$ after blocking PD-1/ PDL1 signaling. In this study, we demonstrated that PDL1 blockade affected neither the ratio of $\mathrm{Tfh} / \mathrm{Tfr}$ cells nor the maturation and differentiation of B cells. Despite the opposing roles of $\mathrm{Tfh}$ and Tfr cells on humoral responses, PD-1 is highly expressed on both of them ${ }^{73}$. In addition, the PD-1/PDL1 signaling exerts inhibitory effects on their proliferation and differentiation, and both Tfh and Tfr cells expand when the pathway is prevented $^{50,74}$. Regarding the divergent findings about the effects of PD-1/PDL1 blockage on humoral responses, we are inclined of the view that PD-1/ PDL1 signaling may play a context-dependent role in humoral immune responses ${ }^{20}$.

Besides the old concept that B cells participate in fetalmaternal immune tolerance via attenuating B-cell capabilities characterized by diminished immune responses and reduced auto-antibodies, recent studies have demonstrated that the increased regulatory B cells (Bregs) play an indispensable role in preventing semi-allogeneic rejection and promoting a stable tolerant microenvironment during normal pregnancy ${ }^{75-78}$. Of particular note, Tfr cells not only regulate GC reaction by inhibiting B-cell maturation, differentiation and antibody production, but also provide IL-10 for Breg-cell proliferation and differentiation ${ }^{79,80}$. Our findings showed that $\mathrm{Tfr}$ cells were consumingly accumulated in the uterus during early-mid gestation. Thus, we speculated that these Tfr cells could limit excessive auto-antibodies produced by B cells, and stimulate the generation of Bregs by providing IL-10, which contributed to the maintenance of normal pregnancy. As mentioned above, previous reports have revealed that blockade of PDL1 signaling results in increased fetal rejection during murine allognenic pregnancy but not syngeneic pregnancy, and this rejection depends on $\mathrm{T}$ cells but not $\mathrm{B}$ cells and $\mathrm{APCs}^{52}$. In addition, deficiency of PDL1 leads to an expansion of Th1, Th17 and Tfh cells but a suppression of Treg and Th2 cells $^{10,12,13,52}$. In this study, we found PDL1 blockage increased fetal resorption and Tfr cells. Nevertheless, we consider the augmented fetal resorption rate and the disordered immune tolerance status not only as a consequence of Tfr cell expansion, but also as a result of the imbalanced Th cell differentiation: in favor of Th1, Th17, and Tfh cells while against the development of Th2 and Treg cells. Overall, we proposed a new Th1/Th2/Th17/ Treg/Tfh/Tfr paradigm during a healthy pregnancy that any of the participants play an important role in maintaining the immune tolerance at fetal-maternal interface during healthy pregnancy.
In summary, we have first presented a previously unknown correlation of $\mathrm{Tfr}$ cells accumulation with healthy allogeneic pregnancy and PDL1 blockade-induced miscarriage. We demonstrate that $\mathrm{CD} 4^{+} \mathrm{CXCR} 5^{\text {hi }}$ PD- ${ }^{\text {hi }}{ }^{\text {Foxp }} 3^{+}$Tfr cells are preferentially enriched in the uterus at mid-gestation and display a distinct phenotype. In addition, increased fetal resorption induced by PDL1 absence has a strong correlation with excessive Tfr cells infiltration and upregulated PD-1 expression on these cells. However, there is no significant change in the Tfh/ Tfr ratio as well as the maturation and differentiation of $B$ cells after PDL1 blockade. Collectively, these findings update our knowledge of the orchestrated immunological alternations during normal pregnancy and provide insightful ideas into the pathogenesis of abortion. Moreover, our data indicate that although antibodies that block PD-1/PDL1 checkpoint pathway including anti-PD-1 and anti-PDL1 are proving to be an effective immunotherapy in some tumors with striking clinical trial results ${ }^{29-31}$, the reproductive safety must be considered when these are used in pregnancy because of the increased inhibitory $\mathrm{Tfr}$ cells and fetal losses in PDL1 blockade mice.

\section{Acknowledgements \\ We thank Wenwen Liu (Shanghai Institute of Immunology, Institute of Medical Sciences, Shanghai Jiaotong University School of Medicine) for her assistance with flow cytometry. This work was supported by the National Key Research and Development Program of China (grant number: 2018YFC1002802 and 2018YFC1004602), the National Natural Science Foundation of China (81974243, 81601291, 81971403 and 81125004), Shanghai Science and Technology Commission (18411963200 and 17411972700), the Chinese Medical Association of Clinical Medicine Special Funds for Scientific Research Projects (17020400709), the Clinical Research Fund of the Internal Peace Maternity and Child Health Hospital (GFY5816 and GFY5820) and the Science and Technology Foundation of Shanghai Jiao Tong University School of Medicine (19XJ11013).}

\section{Author contributions}

W.Z. designed and performed most of the experiments. S.Q. and W.Z. drafted the paper. S.Q., R.W., Y.Z., X.M., F.T., X.R.L. and X.Q. performed a part of the experiments. S.L., L.S. and Y.L. supervised the study, revised and proofread the paper.

\section{Conflict of interest}

The authors declare that they have no conflict of interest.

\section{Publisher's note}

Springer Nature remains neutral with regard to jurisdictional claims in published maps and institutional affiliations.

Supplementary Information accompanies this paper at (https://doi.org/ 10.1038/s41419-020-2313-7).

Received: 12 September 2019 Revised: 30 January 2020 Accepted: 31 January 2020

Published online: 12 February 2020

\footnotetext{
References

1. Negishi, Y., Takahashi, H., Kuwabara, Y. \& Takeshita, T. Innate immune cells in reproduction. J. Obstet. Gynaecol. Res 44, 2025-2036 (2018).
} 
2. Schumacher, A., Sharkey, D. J., Robertson, S. A. \& Zenclussen, A. C. Immune cells at the fetomaternal interface: how the microenvironment modulates immune cells to foster fetal development. J. Immunol. 201, 325-334 (2018).

3. PrabhuDas, M. et al. Immune mechanisms at the maternal-fetal interface: perspectives and challenges. Nat. Immunol. 16, 328-334 (2015).

4. Arck, P. C. \& Hecher, K. Fetomaternal immune cross-talk and its consequences for maternal and offspring's health. Nat. Med 19, 548-556 (2013).

5. Sharpe, A. H. \& Pauken, K. E. The diverse functions of the PD1 inhibitory pathway. Nat. Rev. Immunol. 18, 153-167 (2018).

6. Francisco, L. M., Sage, P. T. \& Sharpe, A. H. The PD-1 pathway in tolerance and autoimmunity. Immunol. Rev. 236, 219-242 (2010).

7. Ghiotto, M. et al. PD-L1 and PD-L2 differ in their molecular mechanisms of interaction with PD-1. Int Immunol. 22, 651-660 (2010).

8. Catakovic, K., Klieser, E., Neureiter, D. \& Geisberger, R. T cell exhaustion: from pathophysiological basics to tumor immunotherapy. Cell Commun. Signal 15 1 (2017).

9. Meggyes, M., Miko, E., Szigeti, B., Farkas, N. \& Szereday, L. The importance of the PD-1/PD-L1 pathway at the maternal-fetal interface. BMC Pregnancy Childbirth 19, 74 (2019).

10. Habicht, A. et al. A link between PDL1 and T regulatory cells in fetomaternal tolerance. J. Immunol. 179, 5211-5219 (2007).

11. Wang, S. et al. Programmed cell death-1 (PD-1) and T-cell immunoglobulin mucin-3 (Tim-3) regulate CD4+ T cells to induce Type 2 helper T cell (Th2) bias at the maternal-fetal interface. Hum. Reprod. 31, 700-711 (2016).

12. D'Addio, F. et al. The link between the PDL1 costimulatory pathway and Th17 in fetomaternal tolerance. J. Immunol. 187, 4530-4541 (2011).

13. Zeng, W. et al. Characterization of $\mathrm{T}$ follicular helper cells in allogeneic normal pregnancy and PDL1 blockage-induced abortion. Sci. Rep. 6, 36560 (2016).

14. Chung, Y. et al. Follicular regulatory T cells expressing Foxp3 and $\mathrm{BCl}-6$ suppress germinal center reactions. Nat. Med. 17, 983-988 (2011).

15. Linterman, M. A. et al. Foxp3+ follicular regulatory T cells control the germinal center response. Nat. Med. 17, 975-982 (2011)

16. Wollenberg, I. et al. Regulation of the germinal center reaction by Foxp3+ follicular regulatory T cells. J. Immunol. 187, 4553-4560 (2011).

17. Miles, B. \& Connick, E. Control of the germinal center by follicular regulatory T cells during infection. Front Immunol. 9, 2704 (2018).

18. Stebegg, M. et al. Regulation of the germinal center response. Front Immunol. 9, 2469 (2018)

19. Fu, W. et al. Deficiency in $T$ follicular regulatory cells promotes autoimmunity. J. Exp. Med 215, 815-825 (2018).

20. Sage, P. T. et al. Dendritic cell PD-L1 limits autoimmunity and follicular T cell differentiation and function. J. Immunol. 200, 2592-2602 (2018).

21. Wang, $X$. et al. The shifted balance between circulating follicular regulatory $T$ cells and follicular helper T cells in patients with ulcerative colitis. Clin. Sci. 131, 2933-2945 (2017).

22. Liu, C. et al. Increased circulating CD4(+)CXCR5(+)FoxP3(+) follicular regulatory $\mathrm{T}$ cells correlated with severity of systemic lupus erythematosus patients. Int. Immunopharmacol. 56, 261-268 (2018).

23. Niu, Q. et al. Enhanced IL-6/phosphorylated STAT3 signaling is related to the imbalance of circulating $T$ follicular helper/T follicular regulatory cells in patients with rheumatoid arthritis. Arthritis Res. Ther. 20, 200 (2018).

24. Baptista, D., Mach, F. \& Brandt, K. J. Follicular regulatory T cell in atherosclerosis. J. Leukoc. Biol. 104, 925-930 (2018).

25. Cha, Z. et al. The prevalence and function of CD4(+)CXCR5(+)Foxp3(+) follicular regulatory $\mathrm{T}$ cells in diffuse large B cell lymphoma. Int. Immunopharmacol. 161, 132-139 (2018).

26. Mughal, T. I. et al. Recent advances in the genomics and therapy of BCR/ABL1positive and -negative chronic myeloproliferative neoplasms. Leuk. Res 67, 67-74 (2018)

27. Wang, N. et al. The checkpoint regulator SLAMF3 preferentially prevents expansion of auto-reactive B cells generated by graft-vs.-host disease. Front. Immunol. 10, 831 (2019)

28. Wang, $X$. et al. Imbalance of circulating $\mathrm{Tfr} / \mathrm{Tfh}$ ratio in patients with rheumatoid arthritis. Clin. Exp. Med. 19, 55-64 (2019).

29. Topalian, S. L. et al. Safety, activity, and immune correlates of anti-PD-1 antibody in cancer. N. Engl. J. Med. 366, 2443-2454 (2012).

30. Baumeister, S. H., Freeman, G. J., Dranoff, G. \& Sharpe, A. H. Coinhibitory Pathways in Immunotherapy for Cancer. Annu Rev. Immunol. 34, 539-573 (2016).

31. Brahmer, J. R. et al. Safety and activity of anti-PD-L1 antibody in patients with advanced cancer. N. Engl. J. Med. 366, 2455-2465 (2012).
32. Delyea, $\mathrm{C}$. et al. CD71(+) erythroid suppressor cells promote fetomaternal tolerance through Arginase-2 and PDL-1. J. Immunol. 200, 4044-4058 (2018).

33. $\mathrm{Li}, \mathrm{C}$. et al. Proteomic analysis of proteins differentially expressed in uterine lymphocytes obtained from wild-type and NOD mice. J. Cell Biochem. 108, 447-457 (2009)

34. Zeng, W. et al. Long-term exposure to decabrominated diphenyl ether impairs CD8 T-cell function in adult mice. Cell Mol. Immunol. 11, 367-376 (2014).

35. Zeng, W. et al. Distinct transcriptional and alternative splicing signatures of decidual CD4(+) T cells in early human pregnancy. Front Immunol. 8, 682 (2017).

36. Lin, Y., Liang, Z., Chen, Y. \& Zeng, Y. TLR3-involved modulation of pregnancy tolerance in double-stranded RNA-stimulated NOD/SCID mice. J. Immunol. 176, 4147-4154 (2006).

37. Dominguez-Villar, M., Baecher-Allan, C. M. \& Hafler, D. A. Identification of T helper type 1-like, Foxp3+ regulatory T cells in human autoimmune disease. Nat. Med. 17, 673-675 (2011).

38. Wan, Z., Lin, Y., Zhao, Y. \& Qi, H. TFH cells in bystander and cognate interactions with B cells. Immunol. Rev. 288, 28-36 (2019).

39. Qin, L. et al. Insights Into the molecular mechanisms of $T$ follicular helpermediated immunity and pathology. Front Immunol. 9, 1884 (2018).

40. Wallin, E. F. et al. Human T-follicular helper and T-follicular regulatory cell maintenance is independent of germinal centers. Blood 124, 2666-2674 (2014).

41. Sage, P. T. \& Sharpe, A. H. T follicular regulatory cells in the regulation of B cell responses. Trends Immunol. 36, 410-418 (2015).

42. Sage, P. T., Paterson, A. M., Lovitch, S. B. \& Sharpe, A. H. The coinhibitory receptor CTLA-4 controls B cell responses by modulating $T$ follicular helper, $T$ follicular regulatory, and T regulatory cells. Immunity 41, 1026-1039 (2014).

43. Sage, P. T., Tan, C. L., Freeman, G. J., Haigis, M. \& Sharpe, A. H. Defective TFH cell function and increased TFR cells contribute to defective antibody production in aging. Cell Rep. 12, 163-171 (2015).

44. Salek Farrokhi, A., Zarnani, A. H. \& Moazzeni, S. M. Mesenchymal stem cells therapy protects fetuses from resorption and induces Th2 type cytokines profile in abortion prone mouse model. Transpl. Immunol. 47, 26-31 (2018).

45. Piccinni, M. P. et al. T helper cell mediated-tolerance towards fetal allograft in successful pregnancy. Clin. Mol. Allergy 13, 9 (2015).

46. Figueiredo, A. S. \& Schumacher, A. The T helper type 17/regulatory T cell paradigm in pregnancy. Immunology 148, 13-21 (2016).

47. Nurieva, R. I. et al. Bcl6 mediates the development of T follicular helper cells Science 325, 1001-1005 (2009).

48. Johnston, R. J. et al. Bcl6 and Blimp-1 are reciprocal and antagonistic regulators of T follicular helper cell differentiation. Science 325, 1006-1010 (2009).

49. Chowdhury, A. et al. Decreased $T$ follicular regulatory cell/T follicular helper cell (TFH) in simian immunodeficiency virus-infected rhesus macaques may contribute to accumulation of TFH in chronic infection. J. Immunol. 195, 3237-3247 (2015).

50. Sage, P. T., Francisco, L. M., Carman, C. V. \& Sharpe, A. H. The receptor PD-1 controls follicular regulatory $\mathrm{T}$ cells in the lymph nodes and blood. Nat Immunol. 14, 152-161 (2013).

51. Nagamatsu, T., Schust, D. J., Sugimoto, J. \& Barrier, B. F. Human decidual stromal cells suppress cytokine secretion by allogenic CD4+ T cells via PD-1 ligand interactions. Hum. Reprod. 24, 3160-3171 (2009).

52. Guleria, I. et al. A critical role for the programmed death ligand 1 in fetomaternal tolerance. J. Exp. Med 202, 231-237 (2005).

53. Munoz-Fernandez, R. et al. Human decidual stromal cells secrete $C-X-C$ motif chemokine 13, express B cell-activating factor and rescue B lymphocytes from apoptosis: distinctive characteristics of follicular dendritic cells. Hum. Reprod. 27, 2775-2784 (2012)

54. Nhan-Chang, C. L. et al. A role for CXCL13 (BCA-1) in pregnancy and intraamniotic infection/inflammation. J. Matern Fetal Neonatal Med. 21, 763-775 (2008).

55. Kwun, J. et al. Crosstalk between $T$ and $B$ cells in the germinal center after transplantation. Transplant. 101, 704-712 (2017).

56. Wallin, E. F. T Follicular regulatory cells and antibody responses in transplantation. Transplant. 102, 1614-1623 (2018).

57. de Graav, G. N. et al. Follicular T helper cells and humoral reactivity in kidney transplant patients. Clin. Exp. Immunol. 180, 329-340 (2015).

58. Chen, W. et al. Low proportion of follicular regulatory $T$ cell in renal transplant patients with chronic antibody-mediated rejection. Sci. Rep. 7, 1322 (2017).

59. Du, J. et al. Invariant natural killer T cells ameliorate murine chronic GVHD by expanding donor regulatory T cells. Blood 129, 3121-3125 (2017). 
60. Sage, P. T. Preventing antibody-mediated rejection during transplantation: the potential of Tfr cells. Transplant. 102, 1597-1598 (2018).

61. Lu, C. Y., Redline, R. W., Shea, C. M., Dustin, L. B. \& McKay, D. B. Pregnancy as a natural model of allograft tolerance. Interactions between adherent macrophages and trophoblast populations. Transplant. 48, 848-855 (1989).

62. McDonald-Hyman, $C$. et al. Therapeutic regulatory T-cell adoptive transfer ameliorates established murine chronic GVHD in a CXCR5-dependent manner. Blood 128, 1013-1017 (2016).

63. Zhang, M. et al. Inducible T-Cell co-stimulator impacts chronic graft-versushost disease by regulating both pathogenic and regulatory $\mathrm{T}$ cells. Front Immunol. 9, 1461 (2018).

64. Dai, S., Jia, R., Zhang, X., Fang, Q. \& Huang, L. The PD-1/PD-Ls pathway and autoimmune diseases. Cell Immunol. 290, 72-79 (2014).

65. Greenwald, R. J., Freeman, G. J. \& Sharpe, A. H. The B7 family revisited. Annu. Rev. Immunol. 23, 515-548 (2005).

66. Shi, J. et al. PD-1 controls follicular $\mathrm{T}$ helper cell positioning and function. Immunity 49, 264-274, e264 (2018).

67. Velu, V. et al. Enhancing SIV-specific immunity in vivo by PD-1 blockade. Nature 458, 206-210 (2009).

68. Butler, N. S. et al. Therapeutic blockade of PD-L1 and LAG-3 rapidly clears established blood-stage Plasmodium infection. Nat. Immunol. 13, 188-195 (2011).

69. Hams, E. et al. Blockade of B7-H1 (programmed death ligand 1) enhances humoral immunity by positively regulating the generation of $\mathrm{T}$ follicular helper cells. J. Immunol. 186, 5648-5655 (2011).

70. Good-Jacobson, K. L. et al. PD-1 regulates germinal center B cell survival and the formation and affinity of long-lived plasma cells. Nat. Immunol. 11, 535-542 (2010).
71. Hamel, K. M. et al. B7-H1 expression on non-B and non-T cells promotes distinct effects on T- and B-cell responses in autoimmune arthritis. Eur. J. Immunol. 40, 3117-3127 (2010).

72. Kawamoto, S. et al. The inhibitory receptor PD-1 regulates IgA selection and bacterial composition in the gut. Science 336, 485-489 (2012).

73. Wing, J. B. et al. A distinct subpopulation of CD25(-) T-follicular regulatory cells localizes in the germinal centers. Proc. Natl. Acad. Sci. USA 114, E6400-E6409 (2017).

74. Hu, Z. Q. \& Zhao, W. H. Critical role of PD-1/PD-L1 pathway in generation and function of follicular regulatory T cells. Cell Mol. Immunol. 10, 286-288 (2013).

75. Jensen, F., Muzzio, D., Soldati, R., Fest, S. \& Zenclussen, A. C. Regulatory B10 cells restore pregnancy tolerance in a mouse model. Biol. Reprod. 89, 90 (2013).

76. Fettke, F. et al. Maternal and fetal mechanisms of $B$ cell regulation during pregnancy: human chorionic gonadotropin stimulates B cells to produce IL-10 while alpha-fetoprotein drives them into apoptosis. Front Immunol. 7, 495 (2016).

77. Esteve-Sole, A. et al. B Regulatory cells: players in pregnancy and early life. Int. J. Mol. Sci. 19, https://doi.org/10.3390/ijms19072099 (2018).

78. Dutta, S., Sengupta, P. \& Haque, N. Reproductive immunomodulatory functions of B cells in pregnancy. Int. Rev. Immunol. 1-14, https://doi.org/10.1080/ 08830185.2019 .1674299 (2019).

79. Laidlaw, B. J. et al. Interleukin-10 from CD4(+) follicular regulatory T cells promotes the germinal center response. Sci. Immunol. 2, https://doi.org/ 10.1126/sciimmunol.aan4767 (2017).

80. Fazilleau, N. \& Aloulou, M. Several follicular regulatory T cell subsets with distinct phenotype and function emerge during germinal center reactions. Front Immunol. 9, 1792 (2018). 